\title{
GEO-LOCATION BASED AUGMENTED REALITY APPLICATION
}

\author{
Prakhar Saxena ${ }^{1}$ \\ ${ }^{I}$ Graduate, School of Computing Science and Engineering, Galgotias University, U.P, India
}

\begin{abstract}
As the inception of mobile app development began, various applications of Augmented Reality that was once only restricted under the avenue of personal computers extended its domain to mobile applications and currently is used in the wide fields of gaming, education, navigation and so on. The exceptional data processing abilities which is possessed by smartphones in concert with its various services such as Global Positioning System (GPS), wireless networks, and compass have authorized Augmented Reality to find a very fruitful proposition in the wide spectrum of navigation based apps. The objective of the proposed work is to structure and design Geo based Augmented Reality application using GART toolkit. The paper initiates with explaining the various components used in a Geo AR app, exploring and explaining the various structure and features of the app and providing a spotlight on the deficiencies and future scope of the targeted developed app [1].
\end{abstract}

Keywords: Augmented Reality, Global Positioning System, Wireless Network, GART, Geo AR app

\section{INTRODUCTION}

Augmented Reality (AR) is the latest product of the ever developing technology that blends and imposes computer generated animations and graphics over real world constraints [2]. Augmented Reality established itself in the field of mobile technology as it came to be used first in the purpose of displaying and presenting meta-information through virtual graphics on consumer's existing environment through geo-tags, more effectively [3].

One of the primary fields in mobile computation technology where the application of Augmented Reality is quite extensive is navigation mainly in the context of GIS and location based services as it overlays the virtual object on the real life environment and serve as an efficient tool for application and services provided to the user which are dependent on the user's or it's environment position [4].

Many SDKs have emerged in the market for making various navigational apps such as Wikitude, Layer Geo and GART have spring up for mobile phones and various other portable devices for making navigational apps based on Augmented Reality using motion sensors, GPS, accelerometers and digital compass [5].

\section{GENERAL CONCEPT}

\subsection{Geo-Based Augmented Reality}

Geo-based Augmented Reality is the combination of Augmented Reality, Location Based System and GPS giving birth to such an advanced and innovative technology. Its fundamental concept is to retrieve the location of device and superimpose the information on screen about the point of interests. Location Based System and GPS are the two primary concept which relies on geolocation information and the fact is Location Based System uses one or more geolocations such as GPS which gives location whereas LBS (Location based Service) use this location to create value just like in Facebook which stores the user's location once and increment the count after each location tag so that the other users got to know which of his/her friends have been here before [6].

Geolocation is closely related to GPS but a slight distinguishable concept may break them apart. Global Positioning System also known as GPS gives the geographic coordinates whereas geolocation gives the meaningful locations rather than just set of coordinates, for examplestreet address.

To simplify the relationship between all such concepts for better insight, a diagram has been represented below displaying simple dependency links on each other.

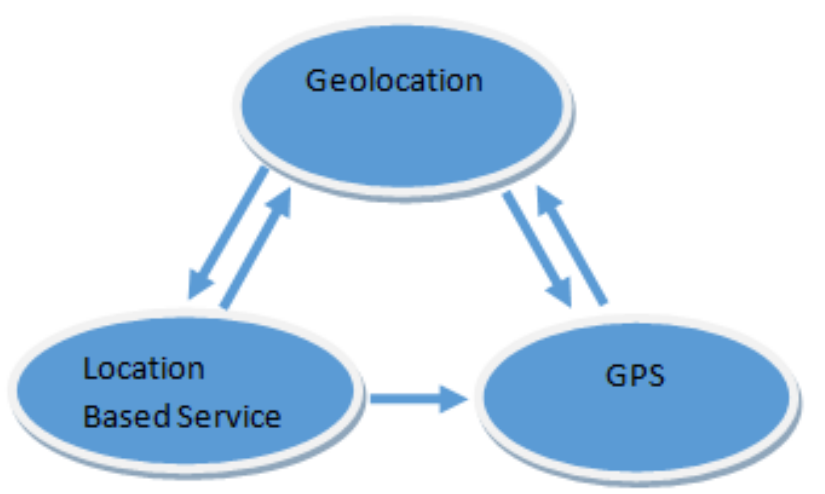

Fig -1: Dependency Diagram Geolocation services

Geo-based AR focuses more on determining the position of an object or person and overlaying the geographical coordinates of the same on screen of dedicated devices in more interactive way rather than just working with positioning services. Next, we will be going to discuss some of the geolocation technologies and service components to work with geographical coordinates before augmenting the information over keen systems. 


\subsection{Few Technologies and Components prior to}

\section{Geolocations}

- GPS: Global positioning system also known as GPS is an essential service component for Geolocation. Its integration into mobile devices has threaten some of the developers and creators for the fact that it may nearly obsolete the use of this technology for specific devices (GPS Navigation). Modern browsers in mobile devices allow to retrieve information from GPS receiver. Greatest coverage, most reliable and more accurate like characteristics made it widely accepted. GPS is a network of 24 satellites placed in a geostationary orbit approx. 20k km from earth [7]. Enabled Synchronized atomic clocks transmits the accurate time and position (longitude, latitude etc.) in space.

- Wi Fi networks: It is an interoperable wireless technology also known as wireless local area network. Components required to access internet over large coverage area are routers incorporated with cable or line modem and $\mathrm{Wi} F i$ access points (basically for homes, organizations, businesses etc.). For cellular carriers subscription to $\mathrm{Wi} F i$, battery-powered routers are used which may include cellular internet radio modem and $\mathrm{Wi} F i$ access points [8]. Its corporation with geolocation helps in determining the exact position through analysing the MAC addresses of the nearby Wi Fi networks. When it combines with GPS, it reloads the database and retrieves the information for different wireless networks. Hybrid system of GPS and Wi Fi networks is potentially the best solution for indoor geolocation where GPS signals are weak [9].

- Compass: Compass is an instrument used for navigational purpose and orientation or direction relative to geographic points. In mobile devices, it is known as magnetometer whose aim is to measure the strength of magnetic field in three dimensions. There is a fact that for augmented reality apps, magnetic interference cause it to behave unpredictably and therefore these sensors are difficult to interpret [10]. In context with geolocation, magnetometer measures earth's magnetic field combined with gravity which is measured by accelerometer to determine the 3-D orientation of phone and that is how it translates the geographical poles and locations and display the contextual information to the user.

\section{SYSTEM DESIGN}

In developing our application for Windows phone, we have used GART [11] (Geo Augmented Reality Toolkit). The major components in the functioning of app are:

- The user

- Camera

- Display Screen

- Mobile GPS

- Augmentation Toolkit

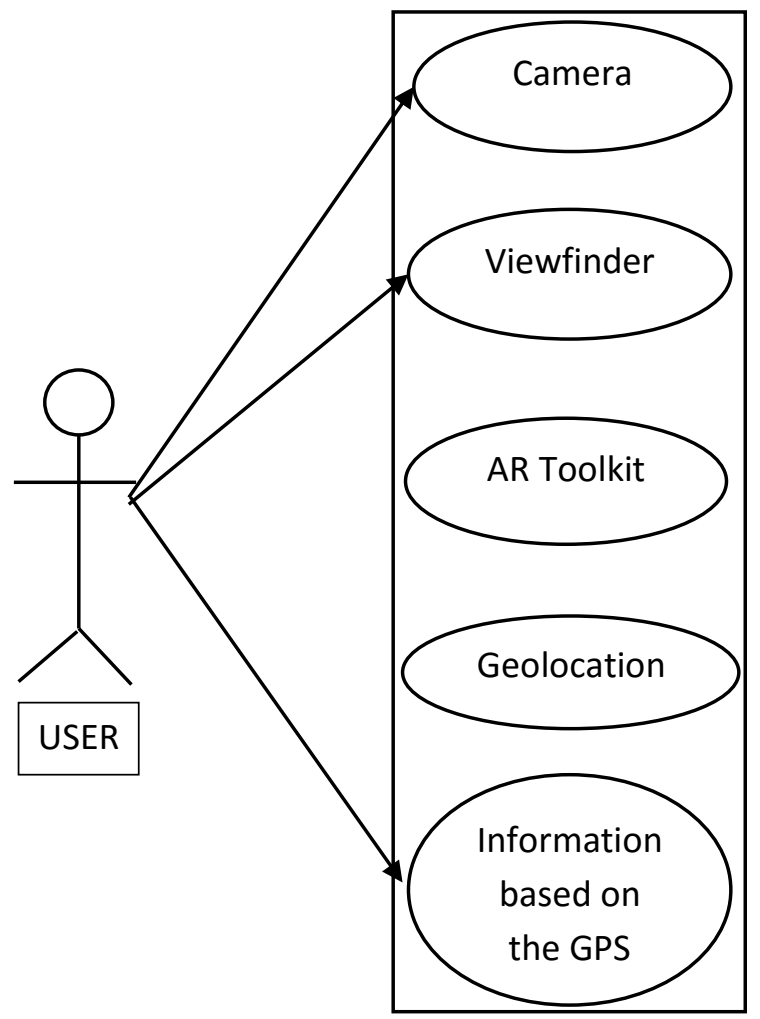

Fig -2: User Interaction with Components in functioning of app

As shown in the above figure, User interacts with the camera and display screen information but has no access to internal GPS or the AR Toolkit (GART).

Figure 3 below shows the step by step procedure along with the time for which each component is active. As we can see that User is active at all the time. User launches the app with activates camera and the display screen. Live feed is taken from camera and Coordinates are matched by the mobile GPS. This information is the input to the AR engine where augmentation is performed and the augmented information is shown on the display screen 


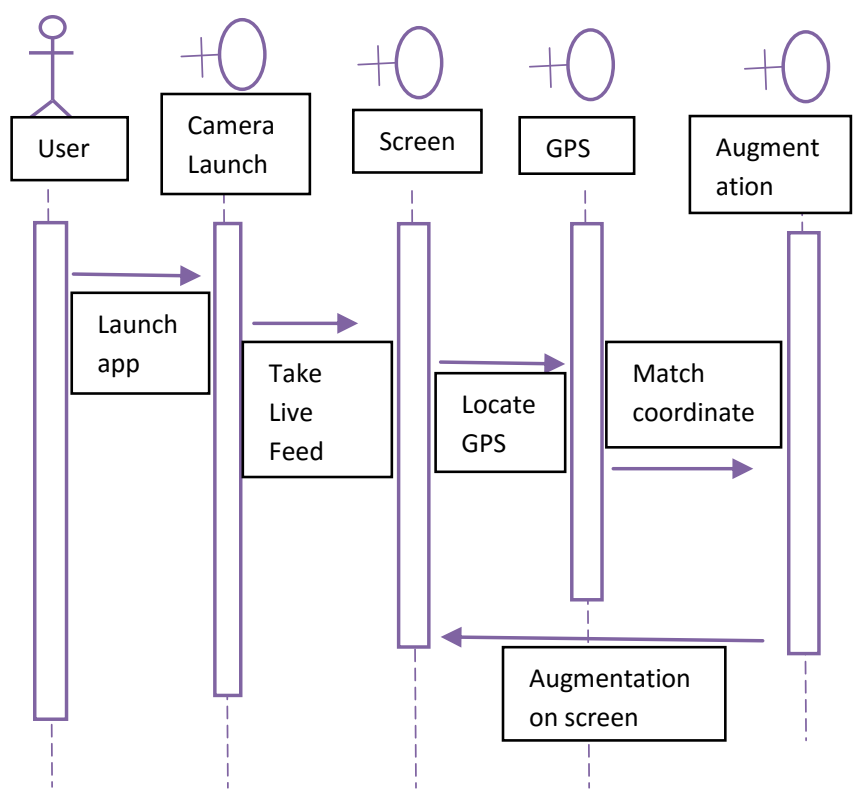

Fig -3: Sequential diagram of each component in app

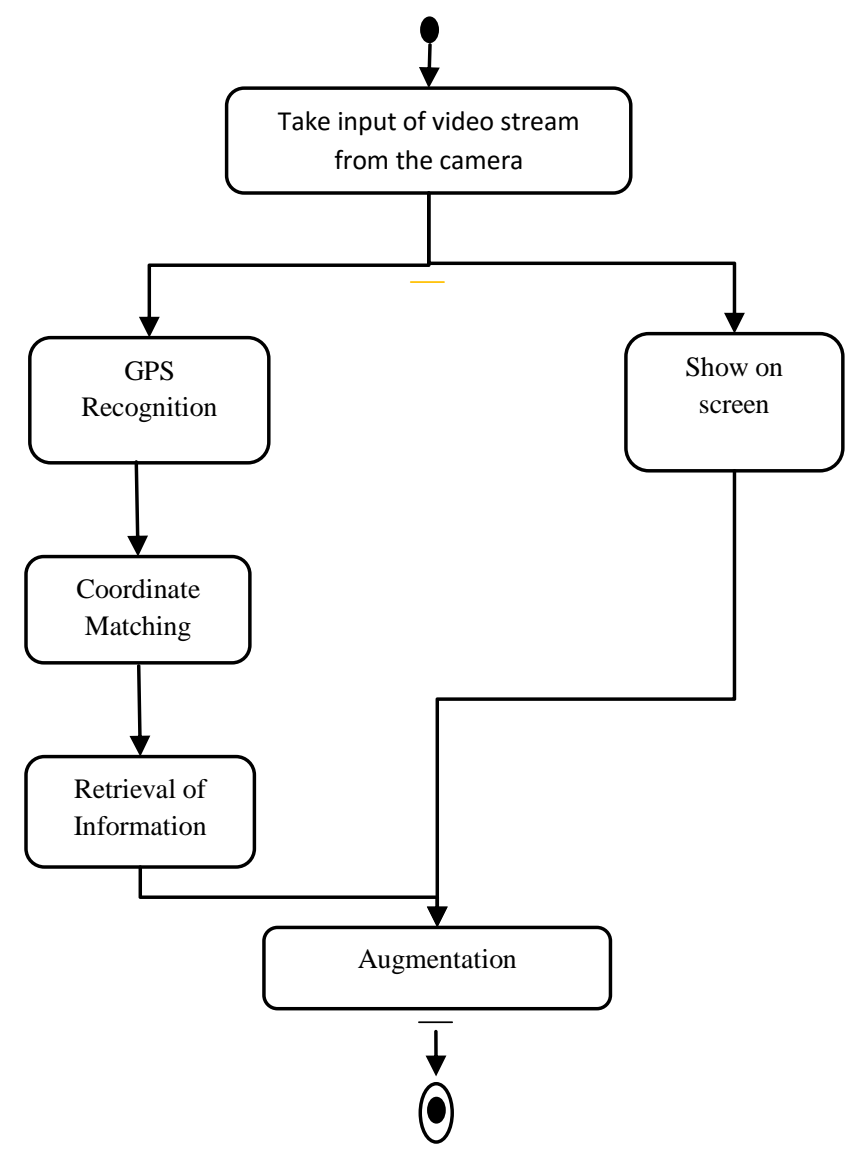

Fig -4: Workflow of Geo-based Augmented Reality app

Shown in figure 4 is the simple workflow diagram which explains that first, the input is taken by AR engine from the camera in the form of live video stream that is shown on the display screen. Then, with the help of mobile GPS, coordinates are matched and the information corresponding to the retrieved coordinates is augmented and shown on the display screen. The main input is the GPS information, not the information from the camera. This is not Marker-based [12] Augmentation hence there is no role of marker.

\section{SDK AND DEVELOPMENT PROCESS}

The Geo-location based AR apps on mobile can be created and developed easily. On comparison with marker-based AR apps where the tiny markers are used for augmentation criteria, in Geo-location based AR apps are less complex and require less computation [13]. We are creating a small Geo-location based AR app on Windows Phone. The main engine we are using in this development is an open source toolkit designed by Jared Bienz of Microsoft Corporation and known as GART Toolkit. The Project is hosted on open source project hosting website codeplex [14]. A brief description of the SDK development technologies and development process is given below.

\subsection{SDK Development Technologies}

- Windows Phone 8 development environment: Windows phone 8 uses .NET framework as defining framework. C\# Programming Language is used as the main programming language of the project [15]. Visual Studio is the main IDE used for development of Windows Phone apps.

- $\quad$ GART Toolkit: GART toolkit is an open source toolkit to write Geo-location based AR apps. By using this we need to provide the app with a collection of objects that have Latitude and Longitude information [16][17]. On the basis of this information and information retrieved by the GPS of smartphone the augmentation is done on the screen of smartphone [18][19].

- Hardware Components required: Augmented Reality apps on smartphone depend heavily on some advanced sensors. Motion Sensor, GPS and a camera is the basic requirement for these apps to work on any Windows Phone powered smartphone [20].

\subsection{Development Process}

- $\quad$ Setting up the environment: We set up the environment by installing the Windows Phone 8 SDK and the project is inspired from the official sample provided by Jared Bienz, the creator of GART Toolkit [21]. The reference to GART.WP8.dll and Microsoft.Phone.Controls.Toolkit.dll are added in the project.

- Designing the User Interface: The user interface of the application has been designed using Extensible Application Markup Language (XAML). The user interface is minimalistic and is inspired from the original sample project provide by Jared Bienz [22]. There is one map control on the main screen which also displays the designated locations on map. When the map mode is turned off the app goes in camera mode and camera starts giving live feed to the GART 
Toolkit and on the main screen. There are options given to user to switch between map mode and camera mode and to augment the feed with location information.

\section{THE CODE BEHIND}

The code behind of the app is programmed in C\# programming language and is very basic and small code. We have an AddLabel method in the mainpage.xaml.cs which is the most important method of the app. In this method the information such as Latitude, Longitude and height information is passed. This information is consolidated in put on screen by AddLabel method [23].

The app uses the motion sensor API [24] of Windows Phone Operating System and motion sensor of smartphone [25] to always show the information which is in front of user's view [26].

\section{CONCLUSION}

In this paper we have explored and evaluated various aspects of augmented reality based Geo-location app used with the help of GART toolkit. The developed app is different from other geo-based apps as rather than superimposing computer-generated virtual graphics on a particular label like other apps which are developed using other SDKs. In this app the virtual data is placed on the existing environment and scenario's by tracing the position and orientation of the portable device user which makes the whole process simple as the need of designing tags is no more needed rather a collection of latitudes and longitudes will serve the same purpose as represented by our app.

\section{ACKNOWLEDGEMENTS}

I would like to thank my supportive friends and researchers Mr Nitish Kumar Sharma, Mr Rohit Kumar Pal and $\mathrm{Mr}$ Anmol Agarwal for being the helping hand during coding part of the app. I would also like to thank my guide Prof. Prabha Nair for inducing zeal and insight of the concepts lying in this work.

\section{REFERENCES}

[1],[2],[3]. Anmol Agarwal, Nitish Kumar Sharma, Piyush Gupta, Prakhar Saxena, Rohit Kumar Pal, Siddharth Mehrotra, Prof. Prabha Nair, Dr. Manoj Wadhwa. Mobile Application Development with Augmented Reality, IJCSE, vol. 2, issue. 5, E-ISSN 2347-2693, May. 2014.

[4]. Sung Hyun Jang (2012). Use-in navigation [Online]. http://issuu.com/gisking/docs/exploring_mobile_augmented _reality_navigation_syst

[5]. Sung Hyun Jang (2012). SDKs [Online]. http://issuu.com/gisking/docs/exploring_mobile_augmented _reality_navigation_syst

[6]. Samuel Charreyron. Different between location based service and global positioning system [Online].

http://www.quora.com/Different-between-location-based-

service-and-global-positioning-system
[7], [9]. Carmen (2013, December 19). Geolocation on mobile device [Online]. http://zeendo.com/info/geolocationon-mobile-device/

[8]. Wi-Fi [Online]. http://en.wikipedia.org/wiki/Wi-Fi

[10]. (2011, July 29). Understanding Smart phone sensor performance: Magnetometer [Online].

http://www.sensorplatforms.com/understanding-smart-

phone-sensor-performance-magnetometer-2/

[11]. Codeplex Open Source Software [Online]. http://gart.codeplex.com/

[12]. Nitish Kumar Sharma, Rohit Kumar Pal, Prakhar Saxena, Prabha Nair (2014). Enactment of AR Based Mobile App in our University. Proceedings of $5^{\text {th }}$ International Conference on Advances in Computer Sciences, Software Solutions, E-learning, Information and Communication Technology (ACSEICT). Held at Jawaharlal Nehru University, New Delhi. October. Journal of Basic and Applied Engineering Research (JBAER). Volume 1 Number 8 pp. 46-49.

[13],[14],[16]. Bienz J. (2013, May). GART Toolkit Project description [Online]. http://gart.codeplex.com/.

[15]. Gallo. Kevin (2012, June 29). Recapping Windows Phone 8 developer News [Online]. http://windowsteamblog.com/windows_phone/b/wpdev/arch ive/2012/06/29/recapping-windows-phone-8-developernews.aspx

[17],[18],[20],[24],[25]. Duncan. G. (2011, October 12). GART-The Geo AR (Augmented Reality) Toolkit for Windows Phone 7.1(5)" [Online]. http://channel9.msdn.com/coding4fun/blog/GART-The-Geo -AR-Augmented-Reality-Toolkit-for-Windows-Phone-715 [19]. Saqib. T. (2014, February 13). Geo Augmented Reality on Your Windows Phone Apps [Online].

http://blogs.msdn.com/b/saqib/archive/2014/02/14/geoaugmented-reality-on-your-windows-phone-apps.aspx [21],[22],[23],[26]. Bienz J. (2014, January 27). GART Toolkit Project Documentation [Online]. http://gart.codeplex.com/documentation

\section{BIOGRAPHY}

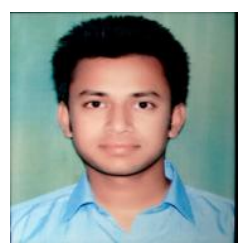

I had pursued Bachelor of Technology in Computer Science from Galgotias University, Greater Noida. My Research interests include Augmented Reality and Mobile application Development. 